\title{
Long-term results for patients with unresectable gastric cancer who received chemotherapy in the Japan Clinical Oncology Group (JCOG) trials
}

\author{
Masana Ohkuwa ${ }^{1,2}$, Atsushi Ohtsu ${ }^{1}$, Narikazu BokU ${ }^{1}$, Shigeaki Yoshida ${ }^{1}$, Yoshinori Miyata ${ }^{3}$, \\ Kuniaki Shirao ${ }^{4}$, Yasuhiro Shimada ${ }^{4}$, and Minoru Kurihara ${ }^{5}$ \\ ${ }^{1}$ Division of Gastrointestinal Oncology / Digestive Endoscopy, National Cancer Center Hospital East, 6-5-1 Kashiwanoha, Kashiwa 277-8577, \\ Japan \\ ${ }^{2}$ Department of Internal Medicine, Ibaraki Prefectural Central Hospital, Ibaraki, Japan \\ ${ }^{3}$ Department of Internal Medicine, Saku Central Hospital, Nagano, Japan \\ ${ }^{4}$ Division of Gastrointestinal Oncology, National Cancer Center Hospital, Tokyo, Japan \\ ${ }^{5}$ Department of Gastroenterology, Showa University Toyosu Hospital, Tokyo, Japan
}

\begin{abstract}
Background. Despite recent developments in chemotherapeutic trials, the long-term results of chemotherapy remain to be clarified. We evaluated the impact of chemotherapy on long-term survival in patients with unresectable gastric cancer. Methods. Between 1985 and 1991, a total of 363 patients with gastric cancer were enrolled into a single randomized phase II study and into three series of phase II studies of the Japan Clinical Oncology Group. The chemotherapy regimens consisted of tegafur + mitomycin C (FTM), uracil-tegafur + mitomycin C (UFTM), 5'deoxy-flurorouridine + cisplatin (5'P), etoposide + doxorubicin + cisplatin (EAP), and 5fluorouracil + cisplatin (FP). After a review of the 363 patients' case records, 226 patients who fulfilled the criteria of having "unresectable" factors prior to chemotherapy became the subjects for this analysis. Of the 226 patients, 50 were in the FTM regimen group, 39, in the UFTM; 49, in the $5^{\prime} \mathbf{P}$; 42, in the EAP; and 46, in the FP group. Survival was updated continually.

Results. Of the 226 patients, $22(10 \%)$ survived longer than 2 years, and 8 (4\%) have survived longer than 5 years. The 85 year survivors consisted of 6 patients who had para-aortic node metastases alone as an "unresectable factor", 1 who had para-aortic and cervical node metastases, and the remaining patient who had liver metastasis alone. Twenty-nine patients with para-aortic node metastasis alone had a significantly longer survival than the other 197 patients $(P<0.001)$.

Conclusion. Systemic chemotherapy may offer some hope of achieving long-term survival in patients with unresectable gastric cancer, particularly when the patient has metastasis only to para-aortic nodes.
\end{abstract}

Key word Gastric cancer · Chemotherapy $\cdot$ Long-term survival

Offprint requests to: A. Ohtsu

Revieved: July 24, 2000 / Accepted: November 7, 2000

\section{Introduction}

Chemotherapy for advanced gastric cancer appears to have a palliative impact. There have been four small studies comparing the outcomes of combination chemotherapy with that of best supportive care [1-4]. In each study, survival was moderately, but significantly, prolonged for the group receiving chemotherapy. Survival rates for patients who received chemotherapy were approximately $40 \%$ at 1 year and $10 \%$ at 2 years, whereas almost all the patients receiving supportive care died within 1 year. Over the past 20 years, many chemotherapeutic agents have been studied in gastric cancer. Although there have been recent reports of very high response rates with some of the newer combination regimens, the median survival of patients with advanced gastric cancer continues to be poor. In each trial involving phase II and phase III studies, survivals were usually evaluated as median survival rates and as 1- or 2-year survival rates. However, there have been few studies that demonstrated the impact of chemotherapy, in terms of long-term survival, in patients with advanced gastric cancer.

The aim of this retrospective analysis was to clarify whether there was an impact of chemotherapy on the long-term survival of patients with unresectable gastric cancer, and to identify the clinical characteristics of the long-term survivors.

\section{Patients and methods}

Between January 1985 and October 1991, a total of 363 patients with histologically confirmed gastric cancer were enrolled into a single randomized phase II study and into three series of phase II studies of the Japan Clinical Oncology Group (JCOG). The chemotherapy 
Table 1. Patient populations in each of the studies

\begin{tabular}{llccc}
\hline Regimen & Accrual period & $\begin{array}{c}\text { No. of patients } \\
\text { accrued }\end{array}$ & $\begin{array}{c}\text { No. of patients } \\
\text { selected }\end{array}$ & Reference \\
\hline FTM & $\begin{array}{c}\text { Jan. 1985-Oct. 1988 } \\
\text { (done as randomized } \\
\text { UhTM }\end{array}$ & 97 & 50 & {$[5]$} \\
& 5hase II study) & & 39 & \\
5'FP & Jan. 1989-Aug. 1990 & 81 & 49 & {$[6]$} \\
EAP & Jun. 1989-Nov. 1990 & 42 & 42 & {$[7]$} \\
FP & Dec. 1990-Oct. 1991 & 57 & 46 & {$[8]$} \\
Total & & 363 & 226 & \\
\hline
\end{tabular}

FTM, Tegafur and mitomycin C; UFTM, uracil-tegafur and mitomycin C; 5'FP, 5'deoxyfluor ouridine and cisplatin; EAP, etoposide, doxorubicin, and cisplatin; FP, 5-fluorouracil and cisplatin ${ }^{a}$ Study compared FTM with UFTM

consisted of the following regimens: tegafur + mitomycin C (FTM) [5], uracil-tegafur + mitomycin C (UFTM) 5), 5'deoxy-flurorouridine + cisplatin $\left(5^{\prime} \mathrm{P}\right)$ [6], etoposide + doxorubicin + cisplatin (EAP) [7], and 5-fluorouracil + cisplatin (FP) [8]. The criteria for patient eligibility in each study varied, particularly for the randomized phase II study comparing FTM with UFTM, which included patients who were medically inoperable with localized disease. To adjust for differences in patient background, the criteria of the EAP study, which were the strictest, were adopted for all 363 patients. The criteria for the EAP study included the following: (i) patients had to have unresectable gastric cancer with histological confirmation, and had to be 70 years or younger, (ii) the patient's performance status (Eastern Cooperative Oncology Group [ECOG] scale) was required to be in the range $0-2$, (iii) patients with moderate or massive ascites or pleural effusion were excluded, and (iv) patients were required to have had no prior treatment (except for surgery), to have no serious complications, and to have adequate bone marrow, liver, and renal functions.

"Unresectable gastric cancer" was defined when the patient had at least one of the following findings: (i) organ metastasis, (ii) distant node metastasis, (iii) peritoneal dissemination detected by barium enema or laparotomy, or (iv) involvement of the adjacent organs confirmed by laparotomy. If there were no obvious descriptions indicating any of the above findings in the case report forms for each study, such cases were excluded from the analysis. From a review of all 363 case record cards, 226 patients were identified as fulfilling these criteria, thereby becoming the subjects of this analysis (Table 1). The 226 patients comprised 50 in the FTM regimen group, 39 in the UFTM, 49 in the 5' P, 42 in the EAP, and 46 patients in the FP group.

The updated survivals of the 226 patients were investigated by each participant investigator. The response results adopted in this analysis were the final results, with extramural review, obtained during each study, which were extracted from the database of each
Table 2. Patient characteristics

\begin{tabular}{|c|c|c|}
\hline \multicolumn{2}{|c|}{ Total no. of patients } & 226 \\
\hline Median & age; years (range) & $60(20-70)$ \\
\hline \multirow[t]{2}{*}{ Sex } & Male & 138 \\
\hline & Female & 88 \\
\hline \multirow[t]{3}{*}{ PS } & 0 & 68 \\
\hline & 1 & 111 \\
\hline & 2 & 47 \\
\hline \multicolumn{3}{|c|}{ Prior gastrectomy } \\
\hline \multicolumn{2}{|l|}{ Yes } & 31 \\
\hline \multicolumn{2}{|l|}{ No } & 195 \\
\hline \multicolumn{3}{|c|}{ Macroscopic type of primary foci } \\
\hline \multicolumn{2}{|c|}{ Expansive } & $57^{\mathrm{c}}$ \\
\hline \multicolumn{2}{|c|}{ Infiltrative } & 164 \\
\hline \multicolumn{3}{|c|}{ Histological type } \\
\hline \multicolumn{2}{|c|}{ Intestinal } & 103 \\
\hline \multicolumn{2}{|c|}{ Diffuse } & 123 \\
\hline \multicolumn{3}{|c|}{ Extent of tumor } \\
\hline \multicolumn{2}{|c|}{ Locally advanced ${ }^{a}$} & 92 \\
\hline \multicolumn{2}{|c|}{ Metastatic } & 134 \\
\hline
\end{tabular}

PS, Performance status (see text for details)

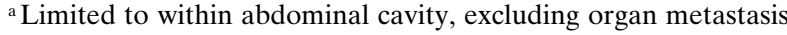

${ }^{\mathrm{b}}$ Organ metastasis and/or any metastasis outside abdominal cavity

${ }^{c}$ Five unknown cases

study. These responses were based on the standard WHO criteria and Japanese response criteria for primary foci [9]. Survival was calculated from the date of initial chemotherapy to death, or to the last follow-up date, using the Kaplan-Meier method [10]. All 226 patients (except for 1 lost to follow-up) were followed for a minimum of 5 years.

\section{Results}

The characteristics of the 226 patients are summarized in Table 2. Most of the patients had a good performance status ( 0 or 1$)$ at the initiation of the chemotherapy. Thirty-one patients had undergone prior gastrectomy. According to histological criteria, 103 patients had intestinal-type and 123 had diffuse-type carcinoma. Eighty-eight patients had peritoneal and/or para-aortic 
node metastases without organ metastasis, and 138 patients had at least one distant metastasis. The response rates in each regimen group were: $8 \%(4 / 50)$ in the FTM regimen group, $21 \%(8 / 39)$ in the UFTM, 35\% $(17 / 49)$ in the 5 'P, $55 \%(23 / 42)$ in the EAP, and $43 \%$ (20/46) in the FP. The median survival time of all 226

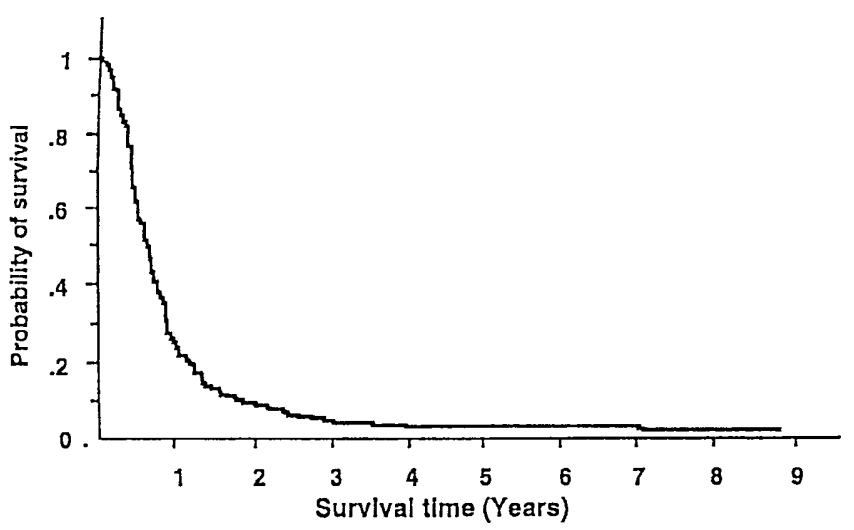

Fig. 1. Overall survival of 226 patients treated with chemotherapy patients was 234 days (Fig. 1). The median survival times for patients in the FTM, UFTM, 5'P, EAP, and FP groups were 183, 217, 243, 297, and 226 days, respectively (Table 3 ).

Of the 226 patients, $22(10 \%)$ survived longer than 2 years, and 8 patients $(4 \%)$ have survived longer than 5 years. The number of 2- and 5-year survivors in each regimen group were: 2 and 0 in the FTM group, 1 and 1 in the UFTM, 8 and 2 in the $5^{\prime} \mathrm{P}, 6$ and 3 in the EAP, and 5 and 2 in the FP groups, respectively. Seventeen $(77 \%)$ of the 22 2-year survivors responded to initial chemotherapy. The 22 2-year survivors comprised 11 patients with para-aortic node metastasis alone as an "unresectable factor", and 11 patients diagnosed as having any distant metastasis whatsoever. All of the 22 patients were classed as PS 0 or 1. Five of the patients had had a gastrectomy before starting chemotherapy. There were no significant differences between histological types in the 2-year survivors.

The characteristics of the eight 5-year survivors are summarized in Table 4. The eight 5-year survivors consisted of six patients with para-aortic node meta-

Table 3. Clinical outcomes of each chemotherapy regimen

\begin{tabular}{lccccc}
\hline Regimen & $\begin{array}{c}\text { No. of } \\
\text { patients }\end{array}$ & $\begin{array}{c}\text { Responders } \\
(\%)\end{array}$ & $\begin{array}{c}\text { MST } \\
\text { (days) }\end{array}$ & $\begin{array}{c}\text { Two-year } \\
\text { survivor }(\%)\end{array}$ & $\begin{array}{c}\text { Five-year } \\
\text { survivors }(\%)\end{array}$ \\
\hline FTM & 50 & $4(8)$ & 183 & $2(4)$ & $0(0)$ \\
UFTM & 39 & $8(21)$ & 217 & $1(3)$ & $1(3)$ \\
5'FP & 49 & $17(35)$ & 243 & $8(16)$ & $2(4)$ \\
EAP & 42 & $23(55)$ & 297 & $6(14)$ & $3(7)$ \\
FP & 46 & $20(43)$ & 226 & $5(11)$ & $2(4)$ \\
Total & 226 & $72(32)$ & 234 & $22(10)$ & $8(4)$ \\
\hline
\end{tabular}

MST, Median survival time

Table 4. Five-year survivors with unresectable gastric cancer

\begin{tabular}{|c|c|c|c|c|c|c|c|c|c|c|c|}
\hline \multirow{2}{*}{$\begin{array}{l}\text { Age } \\
\text { (years) }\end{array}$} & \multirow[b]{2}{*}{ PS } & \multirow[b]{2}{*}{ Macro } & \multirow[b]{2}{*}{ Histology } & \multirow{2}{*}{$\begin{array}{l}\text { Location } \\
\text { of tumor }\end{array}$} & \multirow{2}{*}{$\begin{array}{c}\text { Site of } \\
\text { metastasis }\end{array}$} & \multirow{2}{*}{$\begin{array}{l}\text { Surgical } \\
\text { resection }\end{array}$} & \multirow[b]{2}{*}{ Regimen } & \multicolumn{2}{|c|}{ Response } & \multirow{2}{*}{$\begin{array}{l}\text { Survival } \\
\text { (months) }\end{array}$} & \multirow{2}{*}{$\begin{array}{c}\text { Present } \\
\text { status }\end{array}$} \\
\hline & & & & & & & & $1 \mathrm{st}$ & $2 \mathrm{nd}$ & & \\
\hline 55 & 1 & Infiltrative & Intestinal & A & Liver & - & UFTM & PR & $\mathrm{CR}$ & 73 & Alive: NED \\
\hline 52 & 1 & Infiltrative & Intestinal & A & A-LN & - & 5'FP & $\mathrm{CR}$ & - & 86 & $\begin{array}{c}\text { Dead: cause } \\
\text { unknown }\end{array}$ \\
\hline 62 & 1 & Infiltrative & Intestinal & $\mathrm{CA}$ & A-LN & After ${ }^{a}$ & 5'FP & PR & - & 108 & Alive: NED \\
\hline 57 & 1 & Infiltrative & Diffuse & $\mathrm{C}$ & A-LN & After ${ }^{a}$ & EAP & PR & - & 87 & $\begin{array}{l}\text { Dead: } \\
\text { recurrence }\end{array}$ \\
\hline 53 & 0 & Infiltrative & Diffuse & $\mathrm{FC}$ & $\begin{array}{l}\text { C-LN, } \\
\text { A-LN }\end{array}$ & After $^{\mathrm{a}}$ & EAP & $\mathrm{CR}^{\mathrm{c}}$ & - & 95 & Alive: NED \\
\hline 58 & 0 & Expansive & Intestinal & $\mathrm{C}$ & A-LN & After $^{a}$ & EAP & $\mathrm{CR}^{\mathrm{c}}$ & - & 108 & Alive: NED \\
\hline 47 & 0 & Expansive & Intestinal & FCA & A-LN & Before $^{b}$ & FP & CR & - & 85 & Alive: NED \\
\hline 49 & 0 & Infiltrative & Diffuse & $\mathrm{FC}$ & A-LN & Before $^{b}$ & FP & $\mathrm{NC}$ & CR & 90 & Alive: NED \\
\hline
\end{tabular}

PS, Performance status; Macro, macroscopic type; NED, no evidence of disease; A-LN, paraaortic lymph node (LN); C-LN, cervical LN; F, fundus; C, corpus; A, antrum; after, after chemotherapy; before, before chemotherapy; UFTM; uracil-tegafur and mitomycin C; 5'FP, 5'deoxyflurouridine and cisplatin; EAP, etoposide, doxorubicin, and cisplatin; FP; 5-fluorouracil and cisplatin; CR, complete response; PR, partial response; $\mathrm{NC}$, no change

${ }^{\text {a } A f t e r}$ chemotherapy

${ }^{\mathrm{b}}$ Before chemotherapy

c Pathological CR 
stases alone as an "unresectable factor", one patient with para-aortic and cervical node metastases, and one patient with liver metastasis. Of the eight 5-year survivors, six patients had received surgical resections: two patients had had a gastrectomy before the chemotherapy was initiated, and the other four patients had undergone surgical resections associated with a downgrading of their staging after chemotherapy, including two who were diagnosed with pathological complete remission (CR) in the surgically resected specimen. The remaining two patients did not undergo surgical resection during the follow-up period. One patient (with multiple liver metastases) achieved a CR during the second course of chemotherapy and one patient with para-aortic node metastasis only achieved a CR during the first course of chemotherapy; these two patients were followed-up without additional treatment. All eight of the 5-year survivors presented no evidence of disease at 5 years, while two of the eight patients developed a cancer recurrence after 5 years. Seven of the eight patients achieved objective responses to the initial chemotherapy. Of these seven patients, four achieved a CR after the initial chemotherapy and one patient achieved a CR after the second course of chemotherapy, and two underwent subsequent surgery after succeeding with the initial chemotherapy. One patient, who had not achieved an objective response to the initial chemotherapy (FP), achieved a CR during the third course of chemotherapy, consisting of 5fluorouracil + doxorubicin + mitomycin $\mathrm{C}$.

When patients with unresectable factors were divided into two groups: (i) the 29 patients with para-aortic node metastasis alone and (ii) the remaining 197 patients with other metastases, the former group had a significantly better survival than the latter $(P<0.001$; see Fig. 2). The objective response rate for the 29 patients para-aortic node with metastasis alone was $35 \%(10 / 29)$, which seemed to be comparable to that in the other patients. However, the actual 5-year survival

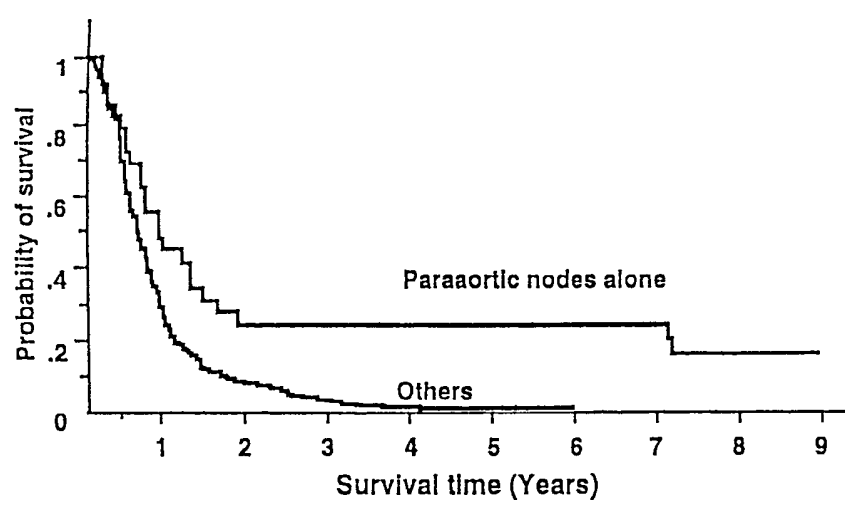

Fig. 2. Survival of 29 patients with para-aortic node metastasis alone (para-aortic nodes alone) compared with survival in the remaining 197 patients (others) rate in patients with para-aortic node metastases alone was significantly better $(24 \%)$ than that in the patients with other metastases $(1 \%)$.

\section{Discussion}

Despite the recent development of new chemotherapeutic agents, unresectable advanced gastric cancer still remains an incurable disease. Some of the recent randomized trials in patients with advanced gastric cancer have revealed significantly, better survival outcomes, compared with either best supportive care alone, or compared with earlier combination regimens. However, these advantages are only marginal, and any improvement in the long-term survival results by these recent treatments still remains unclear. In many phase II and phase III studies for patients with advanced gastric cancer treated with systemic chemotherapy, the median survival times usually ranged from 6 to 10 months, with 2-year survival rates of approximately $10 \%$. The present study also found similar median and 2-year survival rates. Based on these findings, there seem to be no significant differences in survival between Western and Japanese chemotherapeutic trials for advanced disease, in contrast to the significant differences in surgical outcomes for local disease.

There have been a few reports of patients who have survived longer than 5 years with chemotherapy [11,12]. Two reports have been published that indicated 5-year survival rates in patients treated with or without chemotherapy $[13,14]$. However, the results of these reports were too old to compare with the present results, because their data were based on retrospective analyses, using immature staging systems, and there was no precise information on 5-year survivors. In our search of the literature, we found no report indicating a 5-year survival rate for a significant number of patients registered in a prospective study for metastatic gastric cancer and treated with recent systemic chemotherapy. Therefore, the present study is the first report to demonstrate the impact of recent systemic chemotherapy on 5-year survival. Because only 1 of the 226 patients was lost to follow-up, the 5-year survival rate, of $4 \%$, is considered to be equal to the actual survival rate.

It is possible that there was a substantial selection bias in the present study. First, there were some major differences in eligibility criteria between the earlier and later studies. In the earliest study, for instance, patients who were medically inoperable were enrolled, irrespective of whether they had loco-regional disease. Additionally, patients with poor performance status, such as PS 3 on the ECOG scale, were also enrolled. Therefore, we adopted the strictest eligibility criteria, 
which have become the common criteria in modern chemotherapeutic trials, to adjust for subject variability in the studies. Second, the criteria for the descriptor "unresectable" varied between institutions. To exclude patients with a disease that was potentially curable by surgery, "unresectable" factors were defined (as described above in the "Patients and methods" section), and patients without any definite description of "unresectable factor" on their case record cards were excluded from the analysis.

At the time of selection of the subjects, no clinical information or data on survival outcomes had been provided to the investigators. Thus, this selection of the subjects would appear to be directed towards an evaluation of the true impact of systemic chemotherapy in patients enrolled in chemotherapeutic trials for "unresectable" advanced gastric cancer. Of course, these results were obtained from a selective population of patients; namely, those who could be registered in a clinical trial, those who were aged 70 years or younger, those with a good PS, those with adequate organ function, and those with no serious complications.

Another objective of this retrospective study was to clarify the clinical characteristics of the long-term survivors. All eight of the 5-year survivors had a good PS, of 0 or 1 , at entry into the study. No significant differences between histological types were observed. However, six of the eight patients had para-aortic node metastasis alone as an "unresectable factor". We found that there was a marked difference in survival between the 29 patients with para-aortic node metastasis alone and all the other patients. Although most of the paraaortic nodes had not been confirmed histologically, all of the nodes in the 29 patients were a minimum of $1 \mathrm{~cm}$ in size, with a median of $3 \mathrm{~cm}$ in the largest nodes. Therefore, metastases to the para-aortic nodes were relevant, and the above favorable results in this population may demonstrate that such patients with paraaortic node metastasis alone have a greater chance of achieving long-term survival than other patients. Surgical resections were performed in six of the eight 5year survivors, including two patients who had had surgical resections prior to the initial chemotherapy. There are substantial obstacles to overcome in evaluating the role of surgery with respect to long-term survival in advanced gastric cancer. Because indications for surgery, as an adjuvant or palliative treatment, are limited to a very small subpopulation of patients, it is generally difficult to evaluate the role of surgery in clinical trials. In our study, four of the eight 5-year survivors underwent adjuvant surgery following success with chemotherapy that provided long-term survival. These results suggest that adjuvant surgery after chemotherapy with downstaging of the cancer may be an effective approach that is particularly indicated for patients with para-aortic node metastasis alone as an "unresectable factor".

In conclusion, systemic chemotherapy appears to provide some chance of achieving long-term survival in patients with unresectable gastric cancer, particularly when the patients have metastasis exclusively located in the para-aortic nodes.

Acknowledgment This study was supported by a Grant-in-Aid (5S-1) from the Ministry of Health and Welfare of Japan

\section{References}

1. Murad AM, Santiago FF, Petroianu A, Rocha PR, Rodrigues MA, Rausch M. Modified therapy with 5-fluorouracil, doxorubicin, and methotrexate in advanced gastric cancer. Cancer 1993;72:37-41.

2. Glimelius B, Hofman K, Haglund U, Nyren O, Sjoden PO. Initial or delayed chemotherapy with best supportive care in advanced gastric cancer. Ann Oncol 1994;5:189-90.

3. Pyrhonen S, Kuitumen, Kouri M. Randomized comparison of fluorouracil, epidoxorubicin and methotrexate (FEMTX) plus best supportive care with best supportive care alone in patients with non-resectable gastric cancer. Br J Cancer 1995;71:58791

4. Scheithauer W, Kornck G, Zeh B. Palliative chemotherapy versus supportive care in patients with metastatic gastric cancer: a randomized trial (abstract). Second international conference on biology, prevention and treatment of GI malignancy. Cologne, Germany, 1995. p. 68.

5. Kurihara M, Izumi T, Yoshida S, Ohkubo T, Suga S, Kiyohashi A, et al. A cooperative randomized study on tegafur plus mitomycin $\mathrm{C}$ versus combined tegafur and uracil plus mitomycin $\mathrm{C}$ in the treatment of advanced gastric cancer. Jpn J Cancer Res 1991;82:613-20.

6. Koizumi W, Kurihara M, Sasai T, Yoshida S, Morise K, Imamura A, et al. A phase II study of combination therapy with 5'-deoxy5 -fluorouridine and cisplatin in the treatment of advanced gastric cancer with primary foci. Cancer 1993;72:658-62.

7. Shimada Y, Yoshida S, Ohtsu A, Seki S, Saito H. A phase II study of EAP (etoposide, adriamycin and cisplatin) in the patients with advanced gastric cancer: multi-institutional study (abstract). J Jpn Soc Cancer Ther 1991;26:280.

8. Ohtsu A, Shimada Y, Yoshida S, Saito H, Seki S, Morise K, et al. Phase II study of protracted infusional 5-fluorouracil combined with cisplatinum for advanced gastric cancer: report from the Japan Clinical Oncology Group (JCOG). Eur J Cancer 1994; 30A:2091-3.

9. Japanese Research Society for Gastric Cancer. General rules for gastric cancer study (in Japanese). 11th ed. Tokyo: Kanehara; 1985.

10. Kaplan E, Meier P. Nonparametric estimation for incomplete observations. J Am Stat Assoc 1958;53:457-81.

11. Kosaka T, Kayahara M, Yamaguchi A, Yonemura Y, Miwa K, Miyazaki I, et al. A long-surviving case of gastric cancer with liver metastasis without signs of recurrence for 6 years after palliative operation (in Japanese). Gan No Rinsho (Jpn J Cancer Clin) 1987;33:211-6.

12. Wada T, Iwanaga T, Koyama H, Furukawa H, Akita N, Wada A, et al. A case report of long-term survival in advanced gastric cancer with multiple liver metastases treated with mitomycin $\mathrm{C}$ and tegafur therapy after palliative gastrectomy (in Japanese). 
Gan To Kagaku Ryoho (Jpn J Cancer Chemother) 1988;15:3536.

13. Moertel CG. A natural history of advanced gastric cancer. Surg Gynecol Obstet 1968;126:1071-4.
14. Bedikian AY, Chen TT, Khankhanian N, Heibrun LK, McBride $\mathrm{CM}, \mathrm{McMurtrey} \mathrm{MJ}$, et al. The natural history of gastric cancer and prognostic factors influencing survival. J Clin Oncol 1984; 2: 305-10. 